\title{
The Anesthetic Management of Patients Undergoing Nonintubated Video-Assisted Thoracic Surgery
}

\author{
Theresa Gelzinis ${ }^{1}$
}

Accepted: 9 June 2021 / Published online: 16 July 2021

(C) The Author(s), under exclusive licence to Springer Science+Business Media, LLC, part of Springer Nature 2021

\begin{abstract}
Purpose of Review This review focuses on describing the procedural and anesthetic management of patients undergoing nonintubated video-assisted thoracoscopy surgery.

Recent Findings Most thoracic surgery is performed under general endotracheal anesthesia with either a double lumen endotracheal tube or a bronchial blocker. In an attempt to lessen the incidence and severity of postoperative complications, the nonintubated videoassisted thoracoscopic technique was developed, where the surgical procedure is performed under regional anesthesia with sedation. Currently, this technique is recommended for the elderly and in patients with severe cardiopulmonary disease who are at increased risk of complications after general anesthesia. It is the role of the anesthesia team to assist in the decisions whether the patient is a candidate and which block should be performed and to carefully monitor these patients in the operating room.

Summary Nonintubated video-assisted thoracic surgery is an emerging technique with the goal of reducing postoperative complications. The anesthetic technique is highly variable and ranges from general anesthesia with a laryngeal mask airway with a truncal block to thoracic epidural anesthesia with minimal to no block. It is important to have excellent communication with the surgical team and the patient to ensure a safe, successful procedure.
\end{abstract}

Keywords Video-assisted thoracic surgery · Uniportal technique $\cdot$ Thoracic epidural analgesia $\cdot$ Paravertebral block $\cdot$ Intercostal block $\cdot$ Serratus anterior block $\cdot$ Erector spinae plane block $\cdot$ Pendular respiration $\cdot$ Artificial pneumothorax

\section{Introduction}

Upon completion of this lesson, the reader should be able to:

1. Define the concept of nonintubated video-assisted thoracic surgery.

2. Discuss the potential advantages of NIVATS.

3. Consider the patient population that would benefit from the NIVATS technique.

4. Review the patient, anesthetic, and surgical contraindications for NIVATS.

5. Discuss the procedures that can be performed using the NIVATS technique.

6. Discuss the preoperative assessment for NIVATS.

7. Review the anesthetic technique for NIVATS.

8. Review the regional blocks used for NIVATS.

9. Demonstrate the goals of the intraoperative management for patients undergoing the NIVATS procedure.

10. Recognize and treat complications that can occur during NIVATS.

This article is part of the Topical Collection on Thoracic Anesthesia

Theresa Gelzinis gelzinista@upmc.edu

1 Department of Anesthesiology and Perioperative Medicine, University of Pittsburgh, 1328 Fairstead Street, Pittsburgh, PA 15217, USA
Since the mid-2000s, there has been a continuous development of minimally invasive multiportal and uniportal thoracic surgical techniques that allow for more rapid postoperative recovery with reduced morbidity and mortality. Typically, the anesthesia management for patients undergoing thoracic surgery involved general anesthesia with endotracheal intubation, either using a double lumen endotracheal tube or a single lumen tube with a bronchial blocker. With the advent of these new surgical procedures, newer anesthetic techniques have been designed to minimize the perioperative morbidity due to anesthetic management. One technique that has been gaining popularity is the avoidance of intubation by using regional blocks with or without sedation and to minimize the detrimental effects of tracheal intubation and mechanical ventilation, otherwise known as nonintubated video-assisted thoracic surgery (NIVATS).

This technique was first developed by Pompeo et al. in 2004 with video-assisted thoracoscopic (VATS) wedge resections using thoracic epidural anesthesia (TEA) in 
spontaneously breathing patients [1]. As the indications for NIVATS expand and the expertise of the anesthesia and surgical teams increases, NIVATS may quickly become the procedure of choice for multiple thoracic procedures.

\section{Advantages}

The incidence of postoperative pulmonary complications (PPCs), including pneumonia, atelectasis, and acute respiratory distress syndrome, has been estimated to be as high as $37.5 \%$ following thoracic surgery, increasing postoperative morbidity and mortality [2]. General endotracheal anesthesia (GETA) can contribute to the development of PPCs with intubation, mechanical ventilation, and through the use of muscle relaxants and volatile agents $[3 \cdot \bullet]$.

Airway and oropharyngeal trauma can occur during tracheal intubation, especially with the large, rigid double lumen tubes, causing vocal cord and tracheal injuries, hoarseness, as well as dysphagia [4]. Ventilator-induced lung injury, compounded by residual muscle relaxation, can lead to atelectasis, hypoxemia, and the impairment of protective reflexes and [5] volatile agents as well as muscle relaxants and reversal agents can induce nausea and vomiting. Other disadvantages of video-assisted thoracoscopy with general endotracheal anesthesia (VATS-GETA) include compromised cardiac performance and impaired early postoperative respiratory function due to residual paralysis, postoperative pain, nausea, and vomiting and the inability to cough, increasing the risk of pneumonia [6].

The main advantage of NIVATS is the maintenance of spontaneous ventilation, which allows for faster recovery of respiratory muscle function in the early postoperative period, reducing intensive care unit length of stay. The use of intravenous agents for sedation reduces the incidence of postoperative nausea and vomiting. This translates into faster recovery times, reduced costs and hospital length of stay, and lower perioperative morbidity and mortality, especially in the elderly, critically ill, and in high-risk patients with preexisting pulmonary or neuromuscular disease [7]. There is some evidence that patients undergoing NIVATS may have attenuated levels of stress hormones and immunologic responses compared to patients undergoing VATS-GETA [8].

Key Concept Nonintubated video-assisted thoracic surgery avoids complications due to intubation, mechanical ventilation, and muscle relaxants.

\section{Indications and Patient Selection}

Since this technique is constantly evolving, there is no consensus on the optimal candidate for a NIVATS procedure.
Initially, these procedures were performed on low-risk patients for minor thoracic procedures [8]. These included patients with American Society of Anesthesiologists (ASA) class 1 or 2, with a good airway, a body mass index less than 30 , and no significant cardiopulmonary issues $[1,9]$. As expertise in the technique grew, the indications, surgical procedures, and patient populations expanded. Procedures that have been successfully performed using NIVATS include simple procedures such as wedge resections and pleurodesis, to more complex procedures, including lobectomy, thymectomy, carinal, and bronchial resections (Table 1) [10॰]. The patient population has expanded to those in whom the avoidance of intubation and mechanical ventilation is beneficial and includes the elderly and those with severe emphysema, interstitial lung disease, and myasthenia gravis [8]. There appears to be a benefit of NIVATS in high-risk patients, especially with emphysema, where the effect of the surgical pneumothorax on gas exchange and lung function is less pronounced than in lower risk patients [11]. There are a few studies demonstrating the feasibility of NIVATS in small high-risk cohorts [12-14].

\section{Contraindications}

Contraindications to NIVATS can be divided into patient, anesthetic, and surgical contraindications (Table 2). Patient contraindications include hemodynamic instability, noncompliant patients who are unable to tolerate being awake during the procedure, morbid obesity, obstructive sleep apnea, and those with an increased risk for poor airway patency during

Table 1 Procedures performed using NIVATS
1. Pleural/pericardial
a. Pleural biopsy/effusion drainage with or without talc
b. Empyema drainage of empyema
c. Hemothorax evacuation
d. Pericardial window
e. Lung biopsy
f. Treatment of chylothorax
g. Treatment of pneumothorax
2. Lung
a. Biopsy
b. Segmentectomy
c. Lobectomy
d. Lung volume reduction
e. Bronchial resections
f. Carinal resections
g. Tracheal resections
3. Non-lung procedures
a. Mediastinal tumors
b. Thymomas
c. Diaphragmatic plication
d. Esophageal surgery
e. Sympathectomy for palmar hyperhidrosis 
Table 2 Contraindications to NIVATS

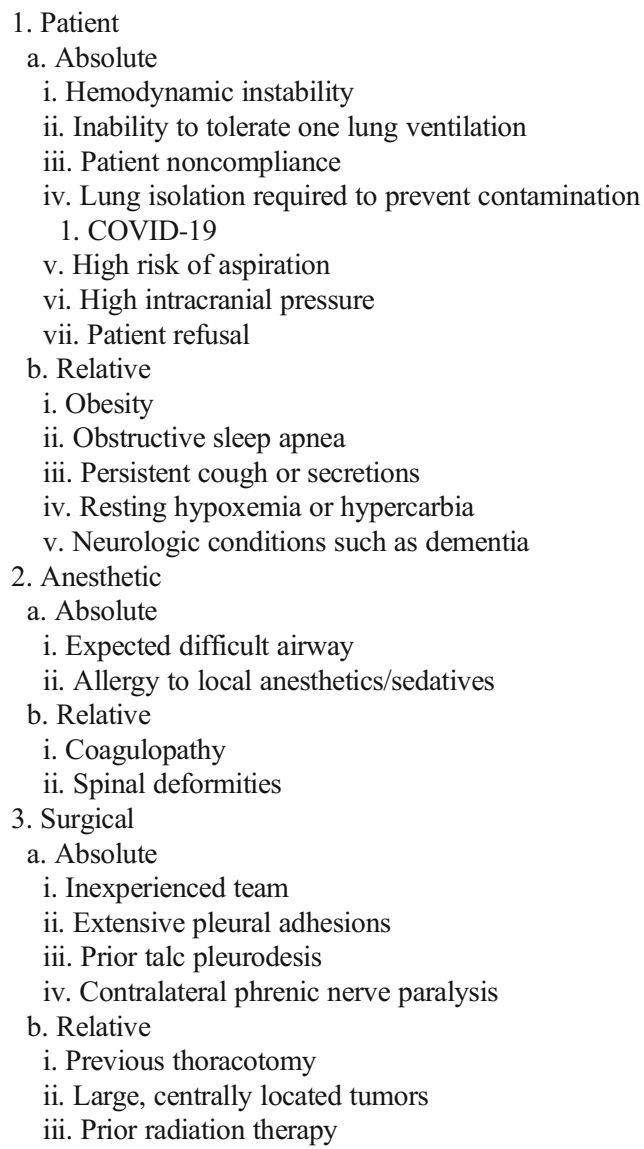

sedation. Other patient contraindications include those who would be intolerant to one lung ventilation and in patients where hypercarbia can be detrimental, including patients with elevated intracranial pressure, uncontrolled systemic hypertension, pulmonary hypertension, and those who are at risk for developing arrhythmias [15]. NIVATS is also contraindicated in patients with infectious diseases that can contaminate the operating room, such as COVID-19. Anesthetic contraindications include patients with an anticipated difficult airway, a coagulopathy with an international normalized ratio (INR) $>1.5$, an allergy to local anesthetics or other medications used in sedation, and neurologic disorders and spinal deformities, which can preclude the performance of neuraxial techniques $[3 \cdot \bullet$ ]. Surgical contraindications to NIVATS include extensive pleural adhesions obliterating the pleural space, previous talc pleurodesis, large centrally located tumors, previous thoracotomy, patients with a high risk of converting to a thoracotomy, and those with prior radiation to the thorax (Table 2).

\section{Preanesthetic Assessment}

The careful selection of patients and an experienced surgical and anesthesiology team are prerequisites for the success of any NIVATS procedure. Besides the typical discussion about the risks and benefits of the procedure, there should be a detailed discussion about what to expect in the operating room, because patient understanding and cooperation are vital for a successful procedure. When evaluating the patient, it is important to perform a thorough airway exam to ensure that the patient can be intubated in the lateral position. The remainder of the preanesthetic assessment should focus on an evaluation of organ systems to determine the suitability for the procedure and for the use of general endotracheal anesthesia, if required. Patients with cardiac or pulmonary diseases should be optimized prior to the surgery. The patient should be able to tolerate one lung ventilation, be cooperative, and be a candidate for a regional anesthesia technique. The patient should not have any of the contraindications listed in Table 2. In addition, the surgical procedure planned should be of a shorter duration.

Key Concept During the preoperative assessment, it is important to determine the eligibility for NIVATS both physically and psychologically. One of the most important assessments is the airway assessment because a difficult airway is a contraindication to NIVATS.

\section{Monitoring}

Monitoring these patients depends on the extent of the resection, the physical status of the patient, and the duration of the surgery. Standard monitors, including noninvasive blood pressure monitoring, electrocardiography, and pulse oximetry, as well as end-tidal carbon dioxide monitoring are mandatory. The use of the bispectral index monitor has been described to assess the depth of sedation [8]. More invasive monitoring, such as intra-arterial and central venous monitoring, is reserved for elderly, frail patients, patients with cardiopulmonary morbidities, or in patients undergoing larger surgical procedures.

\section{Anesthetic Management}

The anesthetic management for NIVATS is just as varied as the indications and surgical techniques themselves. To be successful, adequate analgesia is mandatory and the regional anesthetic technique of choice depends on the extent of the surgery and the expertise of the anesthesia and surgical teams [3••]. For minor procedures with deep sedation, local infiltration at the incision sites is adequate; for uniport procedures, 
truncal blocks, such as the intercostal, erector spinae (ESP), and serratus anterior blocks (SAB), may be sufficient; and in patients undergoing major procedures, especially those who do not tolerate much sedation, neuraxial blocks, such as a paravertebral block (PVB) or thoracic epidural, are essential.

Key Concept It is important to discuss the surgical plan to match the block to the level of sedation and surgical procedure.

\section{Regional Techniques}

\section{Thoracic Epidural Analgesia}

TEA has been considered the gold standard of analgesia after thoracic surgery. Besides postoperative analgesia, the respiratory advantages of TEA include an increased minute ventilation and peak expiratory flow rate, decreased pulmonary vascular resistance, and improved diaphragmatic contractility [16]. TEA also reduces myocardial oxygen demand; improves myocardial blood flow and left ventricular function; and reduces heart rate, arrhythmia formation, and thrombotic complications [17]. The adverse effects of TEA include hypotension, block failure, and postdural puncture headache and when opioids are added, respiratory depression, urinary retention, and pruritus.

\section{Paravertebral Blocks}

The PVB is one of the most frequently used blocks for NIVATS. Local anesthetics are injected into the paravertebral space, a wedged-shaped triangular space adjacent to the thoracic vertebral column. The boundaries of this space are the parietal pleura forming the anterolateral boundary, the base formed by the vertebral body, and the transverse process and the superior costotransverse ligament forming the posterior boundary. The paravertebral space contains adipose tissue, the dorsal ramus, rami communicantes, intercostal nerves and blood vessels, hemiazygos vein, thoracic duct, and sympathetic trunk [18]. Since this space is not covered by the intrathoracic fascia, the nerves are more susceptible to the effects of the local anesthetics.

PVBs have been used as an alternative to TEA because it has been demonstrated to be as effective in providing analgesia with fewer complications, such as hypotension, and may reduce the incidence of postoperative pulmonary complications. Depending on the length and type of procedure, PVBs can be administered as a single shot or as a continuous infusion through a catheter placed percutaneously before or by direct vision intraoperatively. Common complications of this block include block failure, intrathecal or epidural spread of local anesthetics, Horner syndrome, intercostal block, pneumothorax, and vascular puncture [18]. These complications can be reduced with the use of ultrasound. The postoperative benefits of PVBs include a reduction in the incidence of severe vomiting, hypotension, and the need for rescue analgesics and are associated with significantly higher patient satisfaction scores [19].

The use of TEA or PVBs in NIVATS procedures has been reported which includes improved respiratory function, attenuated stress response, and inflammation as measured by lower postoperative white blood cell counts, tumor necrosis factor- $\alpha$, and C-reactive protein levels, improved analgesia, reduced chest drainage, early oral intake, early ambulation, and shortened recovery time [20].

\section{Intercostal Nerve Block}

Intercostal nerve block is another common technique in thoracic surgery. It is simple, safe, and can be done percutaneously or intraoperatively under direct vision. Hsieh et al. retrospectively compared ICNB to no block in 78 patients undergoing uniportal NIVATS for lung resection and found that patients in the ICNB group had lower pain scores, superior performance with incentive spirometry, and consumed less intravenous morphine [21]. The superior analgesia with the ICNB led to shorter chest tube drainage and length of stay [22]. With uniportal NIVATs, the injury is limited to a single intercostal space and a targeted intercostal nerve block at site of the incision has successfully been used for analgesia during NIVATS [23].

\section{Serratus Anterior Block}

The serratus anterior block involves the injection of local anesthetic agents in the plane between the serratus anterior and latissimus dorsi and between the chest wall and the serratus anterior muscles at the level of the 5th rib. This block affects the dermatomal levels corresponding to T2-9 levels [24]. Compared to no block, the SAB was demonstrated to reduce pain and rescue opioids after thoracotomy [25]. There are two reports describing the successful use of SAB for analgesia during NIVATS. Shariat successfully used SAB as a primary anesthetic to drain a pleural effusion via a singleport VATS on a patient receiving antiplatelet agents proving this technique can be used in patients who have a contraindication to TEA and PVB [26]. Corso et al. described the use of the $\mathrm{SAB}$ in a pediatric patient for a NIVATS in a patient with a cardiomyopathy for pleural drainage and biopsy [27]. The SAB appears to be an appropriate block for minor thoracoscopic procedures. 


\section{Erector Spinae Plane Block}

The erector spinae plane block is a paraspinal fascial plane block where under ultrasound guidance, local anesthetic is injected between the erector spinae muscle and the thoracic transverse processes, blocking the dorsal and ventral rami of the thoracic nerves. It has been used successfully for analgesia for a variety of thoracic procedures and a treatment for neuropathic pain following thoracic surgery [28]. $\mathrm{Hu}$ et al. recently described the successful use of the erector spinae plane block in a patient undergoing uniportal NIVATS for bullae resection [29].

\section{Intraoperative Management}

The intraoperative anesthetic management ranges from minimal to no sedation to general anesthesia with an LMA. The anesthetic goals for patients undergoing NIVATS focus on maintaining a patent airway, providing respiratory support, maintaining analgesia and optimizing patient comfort, and dealing with complications, including cough and conversion to GETA $[3 \bullet \cdot$. In patients who require or only tolerate minimal sedation, midazolam with small doses of fentanyl are used. For moderate to deep sedation, a propofol infusion, in combination with remifentanil or dexmedetomidine infusions, is used. The advantage of remifentanil and dexmedetomidine is that they both act as antitussives and have little effect on hypoxic pulmonary vasoconstriction. If a supraglottic device is used, anesthesia may be maintained either with inhalational agents such as sevoflurane or with a total intravenous technique.

Maintaining airway patency in the lateral decubitus position may be challenging with moderate to deep sedation, especially in patients at a higher risk for airway obstruction. Adjuncts to maintain airway patency include nasopharyngeal, oropharyngeal, and laryngeal mask airways. If LMA use is planned, a second- or third-generation LMA can serve as a conduit for bronchoscopy and can facilitate the placement of an airway exchange catheter or endotracheal tube in situations where the conversion to GETA is required. Once the single lumen endotracheal tube is in place, a bronchial blocker can be inserted or the single lumen tube can be exchanged for a double lumen tube. A double lumen tube that would be optimal in this situation is the ANKOR ${ }^{\mathrm{TM}}$ (Insung Med, Wonju, South Korea) double lumen tube. This tube has a carinal cuff between the tracheal and endobronchial cuffs. When the carinal cuff is inflated, it steers the endobronchial cuff into the left mainstem bronchus, allowing proper placement with minimal manipulation.

Methods to improve oxygenation and carbon dioxide removal depend on the mode of airway management. For no to mild sedation, nasal cannula or a simple face mask should be sufficient. For deep sedation or in patients at risk for airway obstruction, hypoxemia, or hypercarbia, the use of a high-flow nasal cannula can be considered. The advantages of a highflow nasal cannula is that high fractions of inspired oxygen can be administered with flow rates up to $60 \mathrm{~L}$ per minute with heated and humidified gas, as well as providing low levels of positive end-expiratory pressure [3・•]. If all else fails, placement of an LMA can be considered. Insertion of an LMA can improve oxygenation by the ability to administer a higher concentration of inspired oxygen, to better monitor end-tidal carbon dioxide and facilitate its removal, and to provide positive pressure ventilation, if necessary.

The conversion to GETA can be risky and there should be plan discussed before the procedure is started. The first decision is whether patients should be turned supine for intubation. Before this occurs, the surgical wound needs to be temporarily draped. Options for airway management in the lateral decubitus position range from fiberoptic or direct laryngoscopic insertion of a single lumen tube or the insertion of a supraglottic airway to facilitate single lumen tube placement. Then one lung ventilation can be initiated after the insertion of a bronchial blocker or an exchange to a double lumen tube. Videolaryngoscopy should be available to improve intubating conditions.

Key Concept It is vital to keep communication open with the surgeon and the operative team and to be prepared to convert to a general endotracheal anesthetic if patient becomes intolerant to the procedure or a complication occurs.

\section{Surgical Technique}

The surgical approach depends on the procedure and the expertise of the surgeon. It is important to determine the approach and the extent of the resection to select the optimal regional anesthetic technique.

\section{Multiportal Approach}

With a multiportal approach for right lung resection, the camera port is inserted at the 4th intercostal space (ICS) along the anterior axillary line, with the operating ports at the 6th ICS along the mid- and posterior axillary line. For a left VATS, the camera port is inserted at the 6th ICS along the posterior axillary line and operating ports at the 6th ICS along the midaxillary line and 4th ICS along the anterior axillary line $\left[30^{\bullet}\right]$. These ports can be shifted one intercostal space depending on the lesion. For thymectomy, the camera port is placed along the upper edge of the 6th ICS in the midaxillary line, with the operative ports inserted into the 3rd ICS along 
the anterior axillary line and into the 6th or 7th ICS along the midclavicular line [30••].

\section{Uniportal Approach}

In an effort to minimize postoperative pain, many NIVATS procedures are performed through a uniportal approach. The first uniportal NIVATS was a lobectomy performed by Gonzalez-Rivas et al., using general anesthesia with a laryngeal mask airway (LMA) [31]. Due to technologic improvements in instrumentation, its use has been steadily increasing and it is the surgical approach of choice for NIVATS. For lung resections, the port is inserted into the 5th ICS slightly anteriorly between the middle and anterior axillary line [32]. For lymph node dissections, and resection of the bronchial sleeve, anterior and middle mediastinal masses, and thymus, the 4th ICS is used [33, 34]. The length of the incision varies from 3 to $6 \mathrm{~cm}$ depending on surgeon preference, tumor size, and the degree of subcutaneous fat [10॰]. When possible, an anterior incision is preferred. With this approach, there is less interference with the intercostal nerve, reducing postoperative pain. Other measures that may reduce postoperative pain include separating the fibers of the serratus anterior muscle instead of cutting into them and by incising the intercostal muscles at the superior rim of the inferior rib to avoid injuring the neurovascular bundle [10•].

Lung inflation following the NIVATS uniportal technique is so complete that no chest tube is required at the end of the surgery, in a procedure called the "tubeless" NIVATS. Cui and his colleagues randomly assigned 173 patients undergoing uniportal NIVATS for a variety of thoracic procedures to either tubeless NIVATS or NIVATS with a postoperative chest tube [35]. They found that the tubeless NIVATS group had more rapid recovery of respiratory muscle function, earlier postoperative oral intake, a lower incidence of systemic complications, reduced inflammatory cytokine levels, decreased incidence of pleural effusion, decreased postoperative infection and pain, and earlier time to postoperative activity and functional exercise. There were no differences between in surgical time, intraoperative blood loss, and perioperative complications. In select populations, patients undergoing minor procedures using tubeless NIVATS have been managed as day surgery patients [6].

Another approach gaining popularity is the subxiphoid uniportal NIVATS, where a 3- to 4-cm incision is made, either vertically just above the xiphoid process and extending down to the end of the process or transversely at the level of the xiphoid process $[36,37]$. The advantages of the subxiphoid approach include reduced access trauma, improved cosmetics, and the ability to access both thoracic cavities, reducing the risk of intercostal nerve injury [38]. The limited incision lends itself to less invasive regional anesthesia techniques, such as the intercostal nerve block (ICNB).

\section{Surgical Technique}

After the patient is placed in the lateral decubitus position, depending on the regional technique used, the surgeon will infiltrate local anesthetic at the incision sites. Preemptive infiltration of the incision site prior to the incision has been related with significantly less postoperative pain. Studies have reported that the presurgical injection of bupivacaine reduced postoperative pain in patients undergoing small procedures such as needlescopic VATS sympathectomy [39, 40]. Single-lung ventilation is achieved by an artificial pneumothorax, created by the equalization of the intrapleural and atmospheric pressures. The lung collapse produced by the surgical pneumothorax is similar to that seen with intubated single-lung ventilation [6]. Hypoxia is less frequent with NIVATS than VATS-GETA due to spontaneous ventilation with efficient diaphragmatic contraction in the lateral decubitus position that optimizes ventilation perfusion matching in the dependent lung. In the majority of procedures, the lung volume will decrease to functional residual capacity, allowing for space for surgical maneuvering. The reduction in oxygenation due to the spontaneous pneumothorax can be corrected with supplemental oxygen. Hypercapnia is also a common finding in these patients. Contributing factors to hypercapnia include prolonged procedure times, shunt due to the partially collapsed lung, hypoventilation from sedation, and rebreathing during pendulum respiration. Pendulum respiration occurs when air from the non-ventilated lung is sucked into the ventilated lung during inspiration and air from the ventilated lung is sucked into the non-ventilated lung during expiration [41]. In these patients, the hypercapnia usually is well tolerated, rarely leads to adverse effects, and usually resolves immediately after surgery.

\section{Complications}

The most common complication that can occur during a NIVATS procedure is cough. The cough reflex is stimulated by the surgical manipulation of the hilum, lung, and bronchi and by increased airway hyper-reactivity as a result of TEAinduced sympathectomy and may interfere with lymph node dissection around the hilum and trachea. This reflex can be abolished by placing topical anesthesia directly onto the surface of the lung as well as by a surgical intrathoracic vagal blockade [42]. Anesthetic adjuncts that may help ameliorate coughing include dexmedetomidine, remifentanil, and sevoflurane. 
Depending on the procedure and the experience of the surgeon, the conversion rate to GETA is between 2.3 and $10 \%$ [41]. Reasons for conversion include patient comfort, a poor surgical field, hypoxia, hypercarbia, hemodynamic instability, and hemorrhage. Patient factors that may result in a conversion to general anesthesia include inadequate analgesia, the feeling of dyspnea due to the pneumothorax, which may lead to anxiety and panic attacks, and intraoperative positioning and immobility, which may not be tolerated in patients with neuromuscular conditions or arthritis [3••]. Surgical factors that may necessitate conversion to GETA include poor maneuverability of instruments due to excessive respiratory movement of the lung and mediastinum, patient movement, pleural adhesions preventing lung collapse, and bleeding. Anesthesia-related factors that require conversion to GETA include intractable hypoxia, hypercarbia, and hemodynamic instability [43]. This is why the anesthesia team has to be vigilant in monitoring these patients.

Key Concept It is important to continuously monitor the patient to rapidly diagnose and treat any potential complications.

\section{Conclusion}

Presently, there are no established indications for the use of NIVATS. The heterogeneity of the surgical and anesthetic techniques and the lack of high-quality randomized controlled trials make it difficult to arrive at any conclusion about patient benefit [3••]. In an attempt to answer this question, several meta-analyses have concluded that patients undergoing NIVATS had shorter procedure times, reduced stress and inflammatory response, improved cellular immune function, shorter periods of postoperative fasting, fewer postoperative complications, shorter chest tube duration, and reduced hospital length of stay but no difference in PPCs or mortality between NIVATS and VATS-GETA [3・•].

Despite the lack of established indications, the recent literature offers evidence of the safety and efficacy of NIVATS. At this time, NIVATS may be beneficial in patients at highrisk for complications with GETA, but more studies are required to further validate both the short-term and long-term outcomes with this procedure [10॰].

\section{Glossary}

ASA

$\mathrm{CM}$

ESPB

GETA

ICNB

ICS

INR

\section{LMA laryngeal mask airway}

NIVATS nonintubated video-assisted thoracic surgery

PPCs

PVB

SAB

TEA

VATS

VATS-

GETA postoperative pulmonary complications

paravertebral block

serratus anterior block

thoracic epidural anesthesia

video-assisted thoracic surgery

video-assisted thoracic surgery under general

endotracheal anesthesia

\section{Declarations}

Conflict of Interest The author does not have any potential conflicts of interest to disclose.

\section{References}

Papers of particular interest, published recently, have been highlighted as:

- Of importance

•- Of major importance

1. Pompeo E, Mineo D, Rogliani P, Sabato AF, Mineo TC. Feasibility and results of awake thoracoscopic resection of solitary pulmonary nodules. Ann Thorac Surg. 2004;78:1761-8.

2. de la Gala F, Piñeiro P, Reyes A, Vara E, Olmedilla L, Cruz P, et al. Postoperative pulmonary complications, pulmonary and systemic inflammatory responses after lung resection surgery with prolonged onelung ventilation. Randomized controlled trial comparing intravenous and inhalational anaesthesia. Br J Anaesth. 2017;119:655-63.

3.• Schieren M, Defosse J. To tube or not to tube: a skeptic's guide to nonintubated thoracic surgery. Curr Opin Anaesthesiol. 2020;34:16. An excellent review of the anesthetic management for NIVATS.

4. Seo J, Cho C, Hong D, et al. The effects of thermal softening of double-lumen endobronchial tubes on postoperative sore throat, hoarseness and vocal cord injuries: a prospective double-blind randomized trial. Br J Anaesth. 2016;116:282-8.

5. Bronsert $\mathrm{M}$, Henderson $\mathrm{W}$, Monk $\mathrm{T}$, et al. Intermediate-acting nondepolarizing neuromuscular blocking agents and risk of postoperative 30-day morbidity and mortality, and long-term survival. Anesth Analg. 2017;124:1476-83.

6. Boisen M, Rao V, Kolarczyk L, et al. The year in thoracic anesthesia: selected highlights from 2016. J Cardiothorac Vasc Anesth. 2017;31:791-9.

7. Al-Abdullatief M, Wahood A, Al-Shirawi N, et al. Awake anaesthesia for major thoracic surgical procedures: an observational study. Eur J Cardiothorac Surg. 2007;32:346-50.

8. Irons J, Martinez G. Anaesthetic considerations for non-intubated thoracic surgery. Journal of Visualized Surgery. 2016;2:61-1.

9. Pompeo E, Tacconi F, Mineo D, Mineo TC. The role of awake video-assisted thoracoscopic surgery in spontaneous pneumothorax. J Thorac Cardiovasc Surg. 2007;133:786-90.

10. Soultanis K, Gonzalez-Rivas D. Devising the guidelines: the concept of uniportal video-assisted thoracoscopic surgery-incisions and anesthetic management. Journal of Thoracic Disease. 2019;11:S2053-61. A good review on the uniportal surgical technique. 
11. Pompeo E. Awake thoracic surgery. In: Pathophysiology of surgical pneumothorax in the awake patient. Bentham Science: Sharja; 2012. p. 9-18.

12. Liang H, Liu J, Wu S, Zhang Y, Liu H, Yang H, et al. Nonintubated spontaneous ventilation offers better short-term outcome for mediastinal tumor surgery. Ann Thorac Surg. 2019;108:1045-51.

13. Jiang L, Depypere L, Rocco G, et al. Spontaneous ventilation thoracoscopic thymectomy without muscle relaxant for myasthenia gravis: comparison with "standard" thoracoscopic thymectomy. J Thorac Cardiovasc Surg. 2018;155:1882-1889.e3.

14. Wang M, Hung M, Hsu H, et al. Non-intubated thoracoscopic surgery for lung cancer in patients with impaired pulmonary function. Annals of Translational Medicine. 2019;7:40-0.

15. Stengl M, Ledvinova L, Chvojka J, Benes J, Jarkovska D, Holas J, et al. Effects of clinically relevant acute hypercapnic and metabolic acidosis on the cardiovascular system: an experimental porcine study. Crit Care. 2013;17:R303.

16. Groeben H. Epidural anesthesia and pulmonary function. J Anesth. 2006;20:290-9.

17. Gruber E, Tschernko E, Kritzinger M, et al. The Effects of thoracic epidural analgesia with bupivacaine $0.25 \%$ on ventilatory mechanics in patients with severe chronic obstructive pulmonary disease. Anesth Analg. 2001:1015-9.

18. Bouman E, Sieben J, Balthasar A, et al. Boundaries of the thoracic paravertebral space: potential risks and benefits of the thoracic paravertebral block from an anatomical perspective. Surg Radiol Anat. 2017;39:1117-25.

19. Wu Z, Fang S, Wang Q, Wu C, Zhan T, Wu M. Patient-controlled paravertebral block for video-assisted thoracic surgery: a randomized trial. Ann Thorac Surg. 2018;106:888-94.

20. Gelzinis T, Sullivan E. Non-intubated general anesthesia for videoassisted thoracoscopic surgery. J Cardiothorac Vasc Anesth. 2017;31:407-8.

21. Hsieh M, Wang K, Liu H, et al. Management of acute postoperative pain with continuous intercostal nerve block after single port videoassisted thoracoscopic anatomic resection. Journal of Thoracic Disease. 2016;8:3563-71.

22. Du K, Wang W, Wang Z. Clinical observation of single-port videoassisted thoracoscopic lobectomy in the treatment of non-small cell lung cancer. Minerva Med:1112020.

23. Gonzalez-Rivas D, Bonome C, Fieira E, et al. Non-intubated videoassisted thoracoscopic lung resections: the future of thoracic surgery? Eur J Cardiothorac Surg. 2015;49:721-31.

24. Blanco R, Parras T, McDonnell J, et al. Serratus plane block: a novel ultrasound-guided thoracic wall nerve block. Anaesthesia. 2013;68:1107-13.

25. Ökmen K, Metin Ökmen B. Evaluation of the effect of serratus anterior plane block for pain treatment after video-assisted thoracoscopic surgery. Anaesthesia Critical Care \& Pain Medicine. 2018;37:349-53.

26. Shariat A, Bhatt H. Successful use of serratus plane block as primary anesthetic for video-assisted thoracoscopic surgery (VATS)assisted pleural effusion drainage. J Cardiothorac Vasc Anesth. 2018;32:e31-2.

27. Corso R, Piraccini E, Byrne H, Poggi P, Tedesco M. The serratus anterior plane block for pediatric non-intubated video-assisted thoracoscopic surgery. Minerva Anestesiol. 2017;83:775-6.

28. Forero M, Adhikary S, Lopez H, et al. The erector spinae plane block. Reg Anesth Pain Med. 2016;41:621-7.

29. Hu ZH, Zou X. The erector spinae plane block (ESPB) for nonintubated video-assisted thoracoscopic surgery. J Clin Anesth. 2019;54:50-1 2019.

30.• Islam A, Mishra R. Evaluation of various port positions for minimal access cardiovascular and thoracic procedures. World J Lap Surg. 2019;12:101-15. An excellent paper describing incision sites for various cardiothoracic procedures.
31. Gonzalez-Rivas D, Fernandez R, de la Torre M, Rodriguez JL, Fontan L, Molina F. Single-port thoracoscopic lobectomy in a nonintubated patient: the least invasive procedure for major lung resection? Interact Cardiovasc Thorac Surg. 2014;19:552-5.

32. Gonzalez-Rivas D, Sihoe A. Important technical details during uniportal video-assisted thoracoscopic major resections. Thorac Surg Clin. 2017;27:357-72.

33. Gonzalez-Rivas D, Yang Y, Stupnik T, et al. Uniportal videoassisted thoracoscopic bronchovascular, tracheal and carinal sleeve resections. Eur J Cardiothorac Surg. 2016;8:S210-22.

34. Sihoe A. Uniportal video-assisted thoracoscopic lobectomy. Annals of Cardiothoracic Surgery. 2016;5:133-44.

35. Cui F, Liu J, Li S, Yin W, Xin X, Shao W, et al. Tubeless videoassisted thoracoscopic surgery (VATS) under non-intubated, intravenous anesthesia with spontaneous ventilation and no placement of chest tube postoperatively. Journal of Thoracic Disease. 2016;8: 2226-32.

36. Suda T, Sugimura H, Tochii D, Kihara M, Hattori Y. Single-port thymectomy through an infrasternal approach. Ann Thorac Surg. 2012;93:334-6.

37. Hernandez-Arenas L, Lin L, Yang Y, et al. Initial experience in uniportal subxiphoid video-assisted thoracoscopic surgery for major lung resections. Eur J Cardiothorac Surg. 2016;50:1060-6.

38. Nan Y, Chu Y, Wu Y, et al. Subxiphoid video-assisted thoracoscopic surgery versus standard video-assisted thoracoscopic surgery for anatomic pulmonary lobectomy. J Surg Res. 2016;200:324-31.

39. Sihoe A, Manlulu A, Lee T, et al. Pre-emptive local anesthesia for needlescopic video-assisted thoracic surgery: a randomized controlled trial. Eur J Cardiothorac Surg. 2007;31:103-8.

40. Fiorelli A, Vicidomini G, Laperuta P, Busiello L, Perrone A, Napolitano F, et al. Pre-emptive local analgesia in video-assisted thoracic surgery sympathectomy is. Eur J Cardiothorac Surg. 2010;37:588-93.

41. Chen J, Cheng Y, Hung M, et al. Nonintubated thoracoscopic lobectomy for lung cancer. Ann Surg. 2011;254:1038-43.

42. Liu J, Cui F, Jianxing H. Non-intubated video-assisted thoracoscopic surgery anatomical resections: a new perspective for treatment of lung cancer. Ann Transl Med. 2015;3:102-7.

43. Shi Y, Yu H, Huang L, Wang S, Chi D, Chen C, et al. Postoperative pulmonary complications and hospital stay after lung resection surgery. Medicine. 2018;97:e10596.

Questions:

1. All of the following anesthetic drugs have antitussive properties except:

a. Sevoflurane

b. Propofol

c. Remifentanil

d. Dexmedetomidine

e. Lidocaine

Answer: B

2. Which surgical procedure is not performed under NIVATS?

a. Lobectomy

b. Lung volume reduction

c. Diaphragmatic plication

d. Lung transplantation

e. Pleural biopsy

Answer: D

3. All of the following are contraindications for NIVATS except:

a. Morbid obesity

b. Previous radiation 
c. Chronic obstructive pulmonary disease

d. Elevated intracranial pressure

e. High risk of converting to thoracotomy

Answer: $\mathrm{C}$

4. Which block is the optimal regional block for a patient undergoing a major procedure but who cannot tolerate sedation?
a. Thoracic epidural
b. Paravertebral
c. Intercostal
d. Serratus anterior
e. Erector spinae

Answer: A

5. What is the conversion rate from NIVATS to general endotracheal anesthesia?
a. $0-1 \%$
b. $1-5 \%$
c. $2-10 \%$
d. $5-15 \%$
e. $10-20 \%$

Answer: C

6. At which intercostal space is the port placed for a uniportal thymectomy?
a. $3^{\text {rd }}$ intercostal space
b. $4^{\text {th }}$ intercostal space
c. $5^{\text {th }}$ intercostal space
d. $6^{\text {th }}$ intercostal space
e. $7^{\text {th }}$ intercostal space
Answer: B

7. The effects of the spontaneous pneumothorax are less pronounced in which disease?

a. Pulmonary fibrosis

b. Myasthenia gravis c. Pulmonary hypertension

d. Spontaneous pneumothorax

e. Emphysema

Answer: E

8. All of the following are true concerning the benefits of NIVATS or VATS-GETA except:

a. Shorter procedure times

b. Reduced incidence of postoperative pulmonary complications

c. Improved cellular immune function

d. Shorter periods of postoperative fasting

e. Reduced hospital length of stay

Answer: B

9. Which regional technique has the added benefit of treating neuropathic pain?
a. Thoracic epidural
b. Paravertebral
c. Serratus anterior
d. Erector spinae
e. Intercostal
Answer: D

10. What is the most common complication during a NIVATS procedure?
a. Severe hypoxia
b. Hemodynamic instability
c. Cough
d. Excessive mediastinal movement
e. Hemorrhage
Answer: C

Publisher's Note Springer Nature remains neutral with regard to jurisdictional claims in published maps and institutional affiliations. 